\title{
A Plasmonic Structure of Fano Resonance in the MIM Waveguide with r-Shaped Resonator for Refractive Index Sensor
}

\section{Siti Rohimah}

Northeast Forestry University

HeTian ( $\nabla$ tianhe@nefu.edu.cn )

Northeast Forestry University https://orcid.org/0000-0002-8325-0851

Jinfang Wang

Shanghai Xing Yue Lian Hui Electronic Technology

Jianfeng Chen

Northeast Forestry University

Jina Li

Northeast Forestry University

Xing Liu

Northeast Forestry University

Jingang Cui

Northeast Forestry University

Qiang Xu

Northeast Forestry University

Yu Hao

Jilin meteorology

\section{Research Article}

Keywords: Surface plasmon polaritons, Fano resonance, r-shaped resonator, Refractive index sensor

Posted Date: January 3rd, 2022

DOI: https://doi.org/10.21203/rs.3.rs-1148319/v1

License: (c) (i) This work is licensed under a Creative Commons Attribution 4.0 International License. Read Full License 


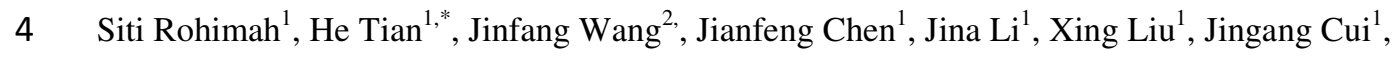

5 Qiang $\mathrm{Xu}^{1}$, and $\mathrm{Yu} \mathrm{Hao}^{3}$

6

$7 \quad{ }^{1}$ College of Science, Northeast Forestry University, Harbin 150040, China

$8 \quad{ }^{2}$ Shanghai Xin Yue Lian Hui Electronic Technology Co. LTD., Shanghai 200233, China

$9 \quad{ }^{3}$ Jilin Meteorological Information Network Center, Changchun 130062, China

10

$11 *$ Corresponding author

12 He Tian, E-mail : tianhe@nefu.edu.cn

13

14 Abstract

15 A plasmonic structure of metal-insulator-metal (MIM) waveguide consisting of a single baffle 16 waveguide and an r-shaped resonator is designed to produce Fano resonance. The finite element 17 method uses the finite element method to analyze the transmission characteristics and magnetic 18 field distributions of the plasmonic waveguide distributions. The simulation results exhibit two 19 Fano resonances that can be achieved by the interference between a continuum state in the baffle 20 waveguide and a discrete state in the r-shaped resonator. The Fano resonances can be simply tuned 21 by changing geometrical parameters of the plasmonic structure. The value variations of 22 geometrical parameters have different effects on sensitivity. Thus, the sensitivity of the plasmonic 23 structure can achieve $1333 \mathrm{~nm} / \mathrm{RIU}$, with a figure of merit of 5876. The results of the designed 24 plasmonic structure offer high sensitivity and nano-scale integration, which are beneficial to 25 refractive index sensors, photonic devices at the chip nano-sensors, and biosensors applications. 
27 Keywords: Surface plasmon polaritons; Fano resonance; r-shaped resonator; Refractive index sensor

29

30 Acknowledgments

31 This work was supported in part by the Fundamental Research Funds for the Central Universities

32 of China (No. 2572020BC08) and in part by the Heilongjiang Provincial Natural Science

33 Foundation of China (No. LH2019F041).

34

35

36

37

38

39

40

41

42

43

44

45

46

47

48

49

50

51

52

53

54 


\section{Introduction}

56 Surface plasmon polaritons (SPPs), which originate from the interaction of incident photons and

57 free electrons on the metal surface, propagate along with the metal-dielectric interface and have

58 the potential to overcome the light diffraction limit, localization of light in subwavelength, and

59 high level of integration capability [1-3]. SPPs have applications in optical devices such as

60 switches [4, 5], sensors [6-9], integrated photonic devices [10], demultiplexers [11], and filters

$61[12,13]$. One of the greatly pledging waveguide structures is the metal-insulator-metal (MIM)

62 waveguide, which has excesses such as low bending loss, simple structure, long propagation

63 distance, deep sub-wavelength confinements, and easy integration [14-18]. Fano resonance is a

64 fundamental resonance phenomenon excited by the interference between a continuum state and a

65 discrete state and typically has sharp resonance peaks and asymmetrical line shapes [19, 20]. Fano

66 resonance can be applied in lasers, slow light devices, and switches [21, 22]. The research

67 developments in Fano resonance based on the MIM waveguide structures have been widely

68 studied and applied to sensors. By way of instance, Wang et al. studied the Fano resonances in

69 MIM waveguide with a baffle and a circular split-ring resonator cavity, and they discussed the

70 refractive index nanosensor with the maximum sensitivity of $1114.3 \mathrm{~nm} / \mathrm{RIU}$ and a figure of merit

71 of 55.71 [18]. Chen et al. designed a MIM waveguide composed of a circular split-ring resonance

72 cavity and a double symmetric rectangular stub waveguide to generate Fano resonance, and the

73 sensitivity was up to $1180 \mathrm{~nm} / \mathrm{RIU}$, and the FOM was 5585.3 [23]. Liu et al. proposed the MIM

74 waveguide structure consisting of a side-coupled rectangular cavity, a rightward opening semi-ring

75 cavity, and a bus waveguide with a silver-air-silver barrier to realize Fano resonances and the

76 sensitivity reached $1550.38 \mathrm{~nm} / \mathrm{RIU}$ [24].

77 This paper designs the plasmonic structure of the MIM waveguide consisting of a baffle 78 waveguide and an r-shaped resonator. The transmission characteristics and magnetic field 79 distributions of the plasmonic structure are investigated using the finite element method (FEM).

80 Two Fano resonances result from the interaction between a continuum state in the baffle

81 waveguide and a discrete state in the $r$-shaped resonator. Then the effects of the geometrical 82 parameters of the plasmonic structure on the Fano resonances are investigated. High sensitivity 83 and figure of merit (FOM) can be achieved by optimizing this structure's parameters. 


\section{Structure Model}

86 The plasmonic structure of the MIM waveguide consists of a single baffle waveguide and an $\mathrm{r}$ -

87 shaped resonator is schematically designed in Fig. 1. The r-shaped resonator is formed of a

88 rectangular resonator and a quarter-ring resonator. The width $W$ of the bus waveguide and $r$-shaped

89 resonator is set at $50 \mathrm{~nm}$ to assure that only the fundamental transverse magnetic mode $\left(\mathrm{TM}_{0}\right)$

90 mode exists in the MIM waveguide [25]. The width of the baffle is $\mathrm{S}$, the coupling distance

91 between the bus waveguide and the $\mathrm{r}$-shaped resonator is $\mathrm{g}$, the height of the rectangular resonator

92 is $\mathrm{H}$, and the effective radius of the quarter-ring resonator is defined as $R^{\prime}=\left(R+R_{0}\right) / 2$, where $R$ and $R_{0}$

93 are the outer and inner radii of the quarter-ring resonator.

95 Fig. 1 The schematic and geometrical parameters of the designed plasmonic structure

In Fig. 1, the blue and the white areas denote silver and air, respectively. Usually, the relative dielectric constant of air is $\varepsilon_{i n}=1$, and the relative dielectric constant of silver is characterized by the Debye-Drude model [26]:

$$
\varepsilon_{m}=\varepsilon_{\infty}-\frac{\omega_{p}^{2}}{\omega^{2}+i \omega \gamma}
$$

where $\varepsilon_{\infty}=3.7$ is the dielectric constant at infinite frequency, $\omega$ is the angular frequency of incident

104 light in vacuum, $\omega_{p}=1.38 \times 10^{16} \mathrm{~Hz}$ is the bulk plasma frequency of free conduction electrons, and $\gamma=2.73 \times 10^{13} \mathrm{~Hz}$ is the electron collision frequency

106 According to the standing wave theoretic, constructive interference should become when the resonance condition is satisfied: $4 \pi R e\left(n_{e f f}\right) l_{1} / \lambda+\phi=2 m \pi, m=1,2,3, \ldots$ [27]. Thus, the resonance wavelength can be derived as $[28,29]$ : 


$$
\lambda=\frac{2 \operatorname{Re}\left(n_{e f f}\right) l_{1}}{m-\phi / 2 \pi}, \quad m=1,2,3, \ldots
$$

111 where $\phi$ is the phase shift introduced by the reflection in the resonance resonator, $l_{1}$ is the length

112 of the resonator, $\mathrm{m}$ is the resonant mode order, and $\operatorname{Re}\left(n_{e f f}\right)$ is the real part of the effective index

$113 n_{\text {eff }}$.

114 The two-dimensional finite element method (FEM) is used to analyze the transmission

115 characteristics of the plasmonic structure. We set the perfect matching layers (PMLs) at the top 116 and bottom of the plasmonic structure to absorb the escaping waves. Light is inputted from Port 1

117 and then outputted from Port 2. Therefore, the transmittance is expressed as $T=\left|S_{21}\right|^{2}$, where $S_{21}$ 118 is the transmission coefficient from Port 1 to Port 2 [30].

\section{Results and Discussions}

121 In this part, Fig. 2 shows the transmission spectra of the single baffle waveguide, the single r122 shaped resonator, and the entire structure that are numerically calculated to investigate the optical 123 properties of the designed structure. The geometrical parameters of the plasmonic structure are set 124 as $\mathrm{R}=200 \mathrm{~nm}, \mathrm{R}_{0}=150 \mathrm{~nm}, \mathrm{H}=300 \mathrm{~nm}, \mathrm{~S}=10 \mathrm{~nm}$, and $\mathrm{g}=10 \mathrm{~nm}$. In Fig. 2, the baffle waveguide 125 produces a continuum state, as indicated by the black line. Meanwhile, the r-shaped resonator 126 coupled with the bus waveguide without the baffle produces discrete states and is marked with two 127 symmetric Lorentzian shapes at $725 \mathrm{~nm}$ and $1275 \mathrm{~nm}$, as described in the red line. As a result, two 128 Fano resonances are obtained by the interference between the continuum state and the discrete 129 state, shown by the blue line in Fig. 2. To simplify the discussion, we name the left Fano 130 resonance as $\mathrm{FR}_{1}$ and the right Fano resonance as $\mathrm{FR}_{2}$. In order to further investigate the two Fano 131 resonances, the magnetic field intensity distributions of the plasmonic structure at $\lambda=720 \mathrm{~nm}\left(\mathrm{FR}_{1}\right.$ 132 peak), $735 \mathrm{~nm}\left(\mathrm{FR}_{1}\right.$ dip), $1265 \mathrm{~nm}\left(\mathrm{FR}_{2}\right.$ peak), and $1305 \mathrm{~nm}\left(\mathrm{FR}_{2}\right.$ dip $)$ are shown in Fig. 3. In Figs. $1333 \mathrm{a}$ and $3 \mathrm{c}$, the magnetic field intensity distributions at Fano resonance peaks are generated owing 134 to the constructive interference between the continuum state and the discrete state and enhanced transmission of the plasmonic structure. However, at Fano resonance dips are generated owing to 
136 the destructive interference [31]. Thus the energy is centralized in the r-shaped resonator and 137 cannot be transmitted through the structure, as shown in Figs. $3 \mathrm{~b}$ and $3 \mathrm{~d}$.

Fig. 2 Transmission spectra of the single baffle waveguide (black line), the single r-shaped resonator (red

Fig. 3 Magnetic field intensity distributions of the plasmonic structure at $\mathbf{a} \lambda=720 \mathrm{~nm}\left(\mathrm{FR}_{1}\right.$ peak), b $\lambda=735$ $\mathrm{nm}\left(\mathrm{FR}_{1} \mathrm{dip}\right)$, $\mathbf{c} \lambda=1265 \mathrm{~nm}\left(\mathrm{FR}_{2}\right.$ peak), and $\mathbf{d} \lambda=1305 \mathrm{~nm}\left(\mathrm{FR}_{2} \mathrm{dip}\right)$

The transmission characteristics of the plasmonic structure can be tuned by varying the parameters. We investigate the effects of the coupling distance $g$ and the baffle width $S$ on the Fano resonances of the plasmonic structure. Fig. 4a describes the transmission spectra of plasmonic structure with different coupling distances $\mathrm{g}$, in which $\mathrm{g}$ is enhanced from $5 \mathrm{~nm}$ to 20 $\mathrm{nm}$ with an interval of $5 \mathrm{~nm}$, and other parameters are set as $\mathrm{R}=200 \mathrm{~nm}, \mathrm{R}_{0}=150 \mathrm{~nm}, \mathrm{H}=300 \mathrm{~nm}$, and $\mathrm{S}=10 \mathrm{~nm}$. The increasing values of $\mathrm{g}$ produce considerable blueshifts of the two Fano resonance wavelengths. The transmission of the Fano resonances gradually decreases when the value of $\mathrm{g}$ increases because the further the r-shaped resonator is from the baffle waveguide, the weaker the coupling strength. Fano resonance wavelength and different values of $g$ have linear relationships, as shown in Fig. 4b. It can be seen that $\mathrm{FR}_{1}$ and $\mathrm{FR}_{2}$ make blueshifts from $710 \mathrm{~nm}$ to $730 \mathrm{~nm}$ and from $1320 \mathrm{~nm}$ to $1235 \mathrm{~nm}$, respectively. Fig. 5a exhibits the transmission spectra of 156 the plasmonic structure with different baffle widths $S$, increasing $S$ from $5 \mathrm{~nm}$ to $20 \mathrm{~nm}$ with an interval of $5 \mathrm{~nm}$, and other parameters are set as $\mathrm{R}=200 \mathrm{~nm}, \mathrm{R}_{0}=150 \mathrm{~nm}, \mathrm{H}=300 \mathrm{~nm}$, and $\mathrm{g}=10 \mathrm{~nm}$.

158 With the increasing values of $\mathrm{S}$, the Fano resonances appear closer to Lorentz resonance, indicating the line shapes becoming sharper and more symmetric. In Fig. 5b, the increasing values of $S$ hardly change the Fano resonance wavelengths. The resonance wavelengths of $\mathrm{FR}_{1}$ and $\mathrm{FR}_{2}$ change slightly from $715 \mathrm{~nm}$ to $720 \mathrm{~nm}$ and from $1260 \mathrm{~nm}$ to $1265 \mathrm{~nm}$, respectively.

Fig. 4 a Transmission spectra of the plasmonic structure with different coupling distances g, b Relationships 
Fig. 5 a Transmission spectra of the plasmonic structure with different baffle widths S, b Relationships between Fano resonance wavelengths and different values of S

Furthermore, the height of rectangular resonator $\mathrm{H}$ and the outer radius of the quarter-ring resonator R are varied. Fig. 6a describes the transmission spectra of the plasmonic structure with

171 different heights of the rectangular resonator $\mathrm{H}$, which $\mathrm{H}$ is enhanced from $290 \mathrm{~nm}$ to $320 \mathrm{~nm}$ with an interval of $10 \mathrm{~nm}$, and other parameters are set as $R=200 \mathrm{~nm}, \mathrm{R}_{0}=150 \mathrm{~nm}, \mathrm{~S}=10 \mathrm{~nm}$, and $\mathrm{g}=10$

$173 \mathrm{~nm}$. The increasing value of $\mathrm{H}$ produces significant redshifts of the Fano resonance wavelengths, and the shift of $\mathrm{FR}_{2}$ is more significant than that of $\mathrm{FR}_{1}$. Relationships between the Fano resonance wavelengths and different values of $\mathrm{H}$ have good linearity, which is indicated by significant redshifts on wavelength peaks of $\mathrm{FR}_{1}$ and $\mathrm{FR}_{2}$ from $710 \mathrm{~nm}$ to $735 \mathrm{~nm}$ and from $1235 \mathrm{~nm}$ to 1325 nm, as shown in Fig. 6b. Transmission spectra of the plasmonic structure for different outer radius $\mathrm{R}$ of the quarter-ring resonator are shown in Fig. 7a. As the increasing value of $\mathrm{R}$, the transmission of $\mathrm{FR}_{1}$ and $\mathrm{FR}_{2}$ gradually decreases, whereas the resonance wavelength of $\mathrm{FR}_{1}$ and $\mathrm{FR}_{2}$ produce obvious redshifts from $700 \mathrm{~nm}$ to $755 \mathrm{~nm}$ and $1225 \mathrm{~nm}$ to $1340 \mathrm{~nm}$ shown in Fig. $7 \mathrm{~b}$. It is also seen that the shift of $\mathrm{FR}_{2}$ is also more significant than that of $\mathrm{FR}_{1}$.

Fig. 6 a Transmission spectra of the plasmonic structure with different heights of rectangular resonator $\mathrm{H}, \mathbf{b}$

Fig. 7 a Transmission spectra of the plasmonic structure with different outer radii R, b Relationships between Fano resonance wavelengths and different values of $R$

Next, we investigate the refractive index sensing based on the effects of the refractive index of the r-shaped resonator on the resonance wavelengths. Generally, sensitivity (S) and figure of merit (FOM) are crucial parameters for assessing the performances of sensors, which are defined as:

$$
S=\frac{\Delta \lambda}{\Delta n},
$$

$$
F O M=\frac{\Delta \mathrm{T}}{T \Delta n}
$$


195 where $\Delta \lambda$ is the resonance wavelength shift, $\Delta n$ is the change of the refractive index, $T$ is the

196 transmittance, and $\Delta T$ is the transmittance change induced by $\Delta n$ at a fixed wavelength [32].

197 The transmission spectra of the plasmonic structure with different refractive indices are shown

198 in Fig. 8a, wherein the refractive index is enhanced from 1.00 to 1.12 with an interval of 0.03 , and

199 the other parameters are set as $R=200 \mathrm{~nm}, \mathrm{R}_{0}=150 \mathrm{~nm}, \mathrm{H}=300 \mathrm{~nm}, \mathrm{~S}=10 \mathrm{~nm}$, and $\mathrm{g}=10 \mathrm{~nm}$. From

200 Fig. 8a, the Fano resonance wavelengths produce significant redshifts with the refractive index

201 increases. Fig. 8b exhibits the linear relationships between the Fano resonance wavelengths and

202 the refractive index. The good linearity of $\mathrm{FR}_{1}$ and $\mathrm{FR}_{2}$ is of great importance for a high-

203 performance sensor. Thus, the sensitivities of the refractive index sensor are about $667 \mathrm{~nm} / \mathrm{RIU}$ for

$204 \mathrm{FR}_{1}$, and $1250 \mathrm{~nm} / \mathrm{RIU}$ for $\mathrm{FR}_{2}$, respectively. Thus, it is known that the sensitivity of $\mathrm{FR}_{2}$ is higher

205 than the sensitivity of $\mathrm{FR}_{1}$.

207 Fig. 8 a Transmission spectra of the plasmonic structure with different refractive indices, b Relationships 208 between Fano resonance wavelengths and different refractive indices

210 Moreover, we investigate the effects of the coupling distance g, the baffle width S, the height 211 of the rectangular resonator $\mathrm{H}$, and the outer radius of the quarter-ring resonator $\mathrm{R}$ on sensitivity.

212 The surrounding refractive index enhances from 1.00 to 1.12 with an interval of 0.03 . Sensitivities

213 of the plasmonic structure on $\mathrm{FR}_{1}$ and $\mathrm{FR}_{2}$ for the different parameters at $\mathrm{g}, \mathrm{S}, \mathrm{H}$, and $\mathrm{R}$ are shown

214 in Fig 9. As observed in Figs. 9a-d, the value variations of g, S, H, and R have different effects on 215 sensitivity. With optimizing geometrical parameters, the maximum sensitivity is $1333 \mathrm{~nm} / \mathrm{RIU}$ at $216 \mathrm{R}=220 \mathrm{~nm}$. Fig. 10 describes the FOM of the plasmonic structure with surrounding refractive 217 index varies from 1.00 to 1.12 with an interval of 0.03 . From the calculation, the obtained FOM is 218 about 5876. The comparison of the sensitivity and FOM between this paper and some other 219 published papers are presented in Table 1. 
Fig. 9 Sensitivities of the plasmonic structure on $\mathrm{FR}_{1}$ and $\mathrm{FR}_{2}$ for the different parameters at a the coupling

Fig. 10 FOM for different refractive indices

Table 1 Comparison the sensitivity and FOM with some other published papers

\begin{tabular}{llll}
\hline Structure & Sensitivity & FOM & Reference \\
\hline $\begin{array}{l}\text { MIM waveguide coupled with } \\
\text { rectangular and ring resonators. }\end{array}$ & $1125 \mathrm{~nm} / \mathrm{RIU}$ & 75 & {$[33]$} \\
$\begin{array}{l}\text { MIM waveguide coupled tangent-ring } \\
\text { resonators. }\end{array}$ & $880 \mathrm{~nm} / \mathrm{RIU}$ & 964 & {$[34]$} \\
$\begin{array}{l}\text { MIM waveguide with a baffle and a } \\
\text { circular split-ring resonator cavity. }\end{array}$ & $1114.3 \mathrm{~nm} / \mathrm{RIU}$ & 55.71 & {$[18]$} \\
$\begin{array}{l}\text { MIM waveguide structures consist of a } \\
\text { cross-shaped cavity and baffle. }\end{array}$ & $1100 \mathrm{~nm} / \mathrm{RIU}$ & $9.95 \times 10^{4}$ & {$[35]$} \\
$\begin{array}{l}\text { MIM waveguide system with } \\
\text { asymmetric X-shaped resonant cavity. }\end{array}$ & $1303 \mathrm{~nm} / \mathrm{RIU}$ & 3113 & {$[10]$} \\
$\begin{array}{l}\text { MIM waveguide with an r-shaped } \\
\text { resonator. }\end{array}$ & $1333 \mathrm{~nm} / \mathrm{RIU}$ & 5876 & This paper \\
\hline
\end{tabular}

228 To prove that the designed plasmonic structure can be applied for biosensors, we study sensing 229 of chemical and biological parameters such as sucrose concentration in a much more exact and 230 efficient manner due to their ability to detect small changes in refractive index. The reason is that 231 the refractive index has unique characteristics for each material [36]. The plasmonic structure can 232 be used for sucrose concentration sensing. The refractive index is taken by ranging from 1.333 to 2331.403 can be gained by varying the sucrose solution concentration [37]. The transmission spectra 234 of the plasmonic structure with different refractive indices of sucrose solution concentrations are 235 shown in Fig. 11a. The refractive index in the r-shaped resonator is enhanced from 1.333 to 1.393 236 with an interval of 0.02. The refractive index can affect the two Fano resonance wavelengths, 237 exhibiting obvious redshifts. From Fig. 11b, the resonance wavelengths and the refractive index 238 have good linearity relationships. As a result, the sensitivities of $\mathrm{FR}_{1}$ and $\mathrm{FR}_{2}$ are about $239675 \mathrm{~nm} / \mathrm{RIU}$ and $1250 \mathrm{~nm} / \mathrm{RIU}$, respectively. The designed plasmonic structure has a useful 240 application in high-sensitivity nanosensors through the proof of refractive indices of sucrose 241 concentration sensing. 
Fig. 11 a Transmission spectra of the plasmonic structure with the different refractive indices of sucrose

\section{Conclusions}

247 This paper designs a plasmonic structure of a MIM waveguide consisting of a baffle waveguide

248 and an r-shaped resonator to produce Fano resonance and investigate its Fano transmission

249 characteristics using the Finite Element Method. Two Fano resonances can be produced and tuned

250 by changing the geometrical parameters of the plasmonic structure. The value variations of

251 coupling distance, baffle width, height of the rectangular resonator, and outer radius of the quarter-

252 ring resonator have different effects on the sensitivity. Thus, the maximum sensitivity can achieve

$2531333 \mathrm{~nm} / \mathrm{RIU}$, with a FOM of 5876. In addition, this plasmonic structure also has the ability of 254 sucrose concentration sensing. Based on these results, the designed plasmonic structure has 255 prospective applications in refractive index nanosensors, nano-photonic devices, and biosensors.

\section{Declarations}

\section{Funding}

260 This work was supported in part by the Fundamental Research Funds for the Central Universities 261 of China (No. 2572020BC08) and in part by the Heilongjiang Provincial Natural Science 262 Foundation of China (No. LH2019F041).

\section{Conflicts of Interest}

265 The authors declare that they have no conflict of interest.

268 Not applicable

270 Code availability 
271 Not applicable

272

273 Author's contributions

274 Siti Rohimah have contributed to Conceptualization, design, analysis, writing, review, and editing.

275 He Tian has contributed to Supervision, Funding acquisition, review, and editing manuscript.

276 Jinfang Wang, Jianfeng Chen, Jina Li, Xing Liu, Jingang Cui, Qiang Xu, and Yu Hao contributed

277 to validating and editing the manuscript. All authors have read and agreed to the published version

278 of the manuscript.

279

280 Ethics approval

281 Not applicable

282

283 Concent to participle

284 Not applicable

285

286 Consent to Publish

287 The manuscript has not been published before and is not being considered for publication

288 elsewhere. All authors have contributed to the manuscript creation and read and approved the final 289 manuscript.

290

291

292

293

294

295 


\section{$301 \quad$ References}

302 1. Barnes WL, Dereux A, Ebbesen TW (2003) Surface plasmon subwavelength optics. Nature 424:824-830. https://doi.org/10.1038/nature01937 Bochenkov VE, Frederiksen M, Sutherland DS (2013) Enhanced refractive index sensitivity of elevated shortrange ordered nanohole arrays in optically thin plasmonic Au films. Opt Express 21(12):14763. https://doi.org/10.1364/oe.21.014763

307 Schuller JA, Barnard ES, Cai W, et al (2010) Plasmonics for extreme light concentration and manipulation. Nat Mater 9:193-204. https://doi.org/10.1038/nmat2630 Yang SL, Yu DM, Liu GD, et al (2018) Perfect Plasmon-Induced Absorption and Its Application for MultiSwitching in Simple Plasmonic System. Plasmonics 13:1015-1020. https://doi.org/10.1007/s11468-017-0599-9 Shahamat Y, Vahedi M (2019) Mid-infrared plasmonically induced absorption and transparency in a Si-based structure for temperature sensing and switching applications. Opt Commun 430:227-233. https://doi.org/10.1016/j.optcom.2018.08.047

Chen J, Sun C, Gong Q (2014) Fano resonances in a single defect nanocavity coupled with a plasmonic waveguide. Opt Lett 39:0146-9592. https://doi.org/10.1364/ol.39.000052 Chen Z, Cui L, Song X, et al (2015) High sensitivity plasmonic sensing based on Fano interference in a rectangular ring waveguide. Opt Commun 340:1-4. https://doi.org/10.1016/j.optcom.2014.11.081 Pang S, Huo Y, Xie Y, Hao L (2017) Tunable Electromagnetically Induced Transparency-Like in Plasmonic Stub Waveguide with Cross Resonator. Plasmonics 12:1161-1168. https://doi.org/10.1007/s11468-016-0371-6

320 9. Xiao G, Xu Y, Yang H, et al (2021) High Sensitivity Plasmonic Sensor Based on Fano Resonance with Inverted U-Shaped Resonator. sensors 21:1164. https://doi.org/10.3390/s21041164

10. Jina L, Chen J, Xing L, et al (2021) Optical sensing based on multimode Fano resonances in metal-insulatormetal waveguide systems with X-shaped resonant cavities. Appl Opt 60:5312-5319

324 11. Rahimzadegan A, Granpayeh N, Hosseini SP (2014) Improved plasmonic filter, ultra-compact demultiplexer, and splitter. J Opt Soc Korea 18:261-273. https://doi.org/10.3807/JOSK.2014.18.3.261

326 12. Khani S, Danaie M, Rezaei P (2019) Design of a Single-Mode Plasmonic Bandpass Filter Using a Hexagonal 
329 13. Janković N, Cselyuszka N (2019) High-Resolution Plasmonic Filter and Refractive Index Sensor Based on Perturbed Square Cavity with Slits and Orthogonal Feeding Scheme. Plasmonics 14:555-560. https://doi.org/10.1007/s11468-018-0834-z

332 14. Veronis G, Fan S (2007) Theoretical investigation of compact couplers between dielectric slab waveguides and two-dimensional metal-dielectric-metal plasmonic waveguides. Opt Express 15:1211-1221. https://doi.org/10.1364/oe.15.001211

Bian Y, Zheng Z, Liu Y, et al (2011) Hybrid wedge plasmon polariton waveguide with good fabrication-errortolerance for ultra-deep-subwavelength mode confinement. Opt Express 19:22417-22422. https://doi.org/10.1364/oe.19.022417

338 16. Jeong CY, Kim M, Kim S (2013) Circular hybrid plasmonic waveguide with ultra-long propagation distance. Opt Express 21:17404-17412. https://doi.org/10.1364/oe.21.017404

18. Nanosensor I, Wang M, Zhang M, et al (2019) Fano Resonance in an Asymmetric MIM Waveguide Structure and Its Application in a Refractive. sensors 19:791-800. https://doi.org/10.3390/s19040791

344 19. Luk'Yanchuk B, Zheludev NI, Maier SA, et al (2010) The Fano resonance in plasmonic nanostructures and metamaterials. Nat Mater 9:707-715. https://doi.org/10.1038/nmat2810 (3):2257-2298. https://doi.org/10.1103/RevModPhys.82.2257 nanostructures. Opt Express 22:29693. https://doi.org/10.1364/oe.22.029693 
365 29. Hu F, Yi H, Zhou Z (2011) Band-pass plasmonic slot filter with band selection and spectrally splitting

366

36730

371

372

373 capabilities. Opt Express 19:4848. https://doi.org/10.1364/oe.19.004848

30. Fang Y, Wen K, Qin Y, et al (2019) Multiple fano resonances in an end-coupled MIM waveguide system. Opt Commun 452:12-17. https://doi.org/10.1016/j.optcom.2019.06.076

31. Liu X, Li J, Chen J, et al (2020) Fano resonance based on D-shaped waveguide structure and its application for human hemoglobin detection. Appl Opt 59:6424-6430. https://doi.org/10.1364/AO.397976

32. Binfeng Y, Hu G, Zhang R, Yiping C (2016) Fano resonances in a plasmonic waveguide system composed of stub coupled with a square cavity resonator. J Opt 18:055002: https://doi.org/10.1088/2040-8978/18/5/055002

33. Tang Y, Zhang Z, Wang R, et al (2017) Refractive Index Sensor Based on Fano Resonances in Metal-InsulatorMetal Waveguides Coupled with Resonators. sensors 17:784-792. https://doi.org/10.3390/s17040784

34. Guo Z, Wen K, Hu Q, et al (2018) Plasmonic multichannel refractive index sensor based on subwavelength tangent-ring metal-insulator-metal waveguide. Sensors (Switzerland) 18:. https://doi.org/10.3390/s18051348

35. Yang Q, Liu X, Guo F, et al (2020) Multiple Fano resonance in MIM waveguide system with cross-shaped cavity. Optik (Stuttg) 220:165163. https://doi.org/10.1016/j.ijleo.2020.165163

Li H, Lin L, Xie S (2000) Refractive index of human whole blood with different types in the visible and nearinfrared ranges. Laser-Tissue Interact XI Photochem Photothermal, Photomech 3914:517. https://doi.org/10.1117/12.388073

37. Chen CH, Tsao TC, Tang JL, Wu W Te (2010) A multi-D-shaped optical fiber for refractive index sensing. Sensors 10:4794-4804. https://doi.org/10.3390/s100504794 


\section{Figures}

\section{Figure 1}

The schematic and geometrical parameters of the designed plasmonic structure

\section{Figure 2}

Transmission spectra of the single baffle waveguide (black line), the single r-shaped resonator (red line), and the entire structure (blue line)

\section{Figure 3}

Magnetic field intensity distributions of the plasmonic structure at $\mathbf{a} \lambda=720 \mathrm{~nm}\left(\mathrm{FR}_{1}\right.$ peak), $\mathbf{b} \lambda=735 \mathrm{~nm}$ (FR 1 dip), $\mathbf{c} \lambda=1265 \mathrm{~nm}\left(\mathrm{FR}_{2}\right.$ peak), and $\mathbf{d} \lambda=1305 \mathrm{~nm}\left(\mathrm{FR}_{2} \mathrm{dip}\right)$

\section{Figure 4}

a Transmission spectra of the plasmonic structure with different coupling distances $\mathrm{g}$, b Relationships between Fano resonance wavelengths and different values of $g$

\section{Figure 5}

a Transmission spectra of the plasmonic structure with different baffle widths S, b Relationships between Fano resonance wavelengths and different values of $S$ 

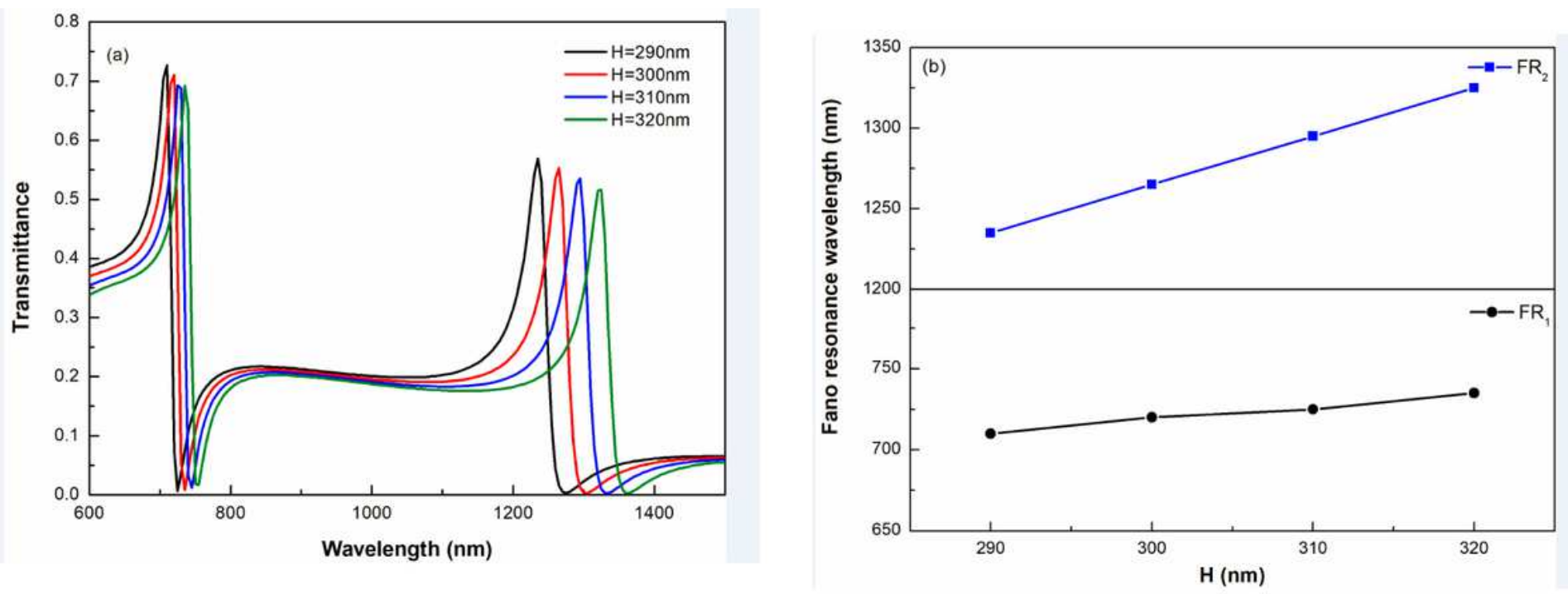

Figure 6

a Transmission spectra of the plasmonic structure with different heights of rectangular resonator $\mathrm{H}, \mathbf{b}$ Relationships between Fano resonance wavelengths and different values of $\mathrm{H}$
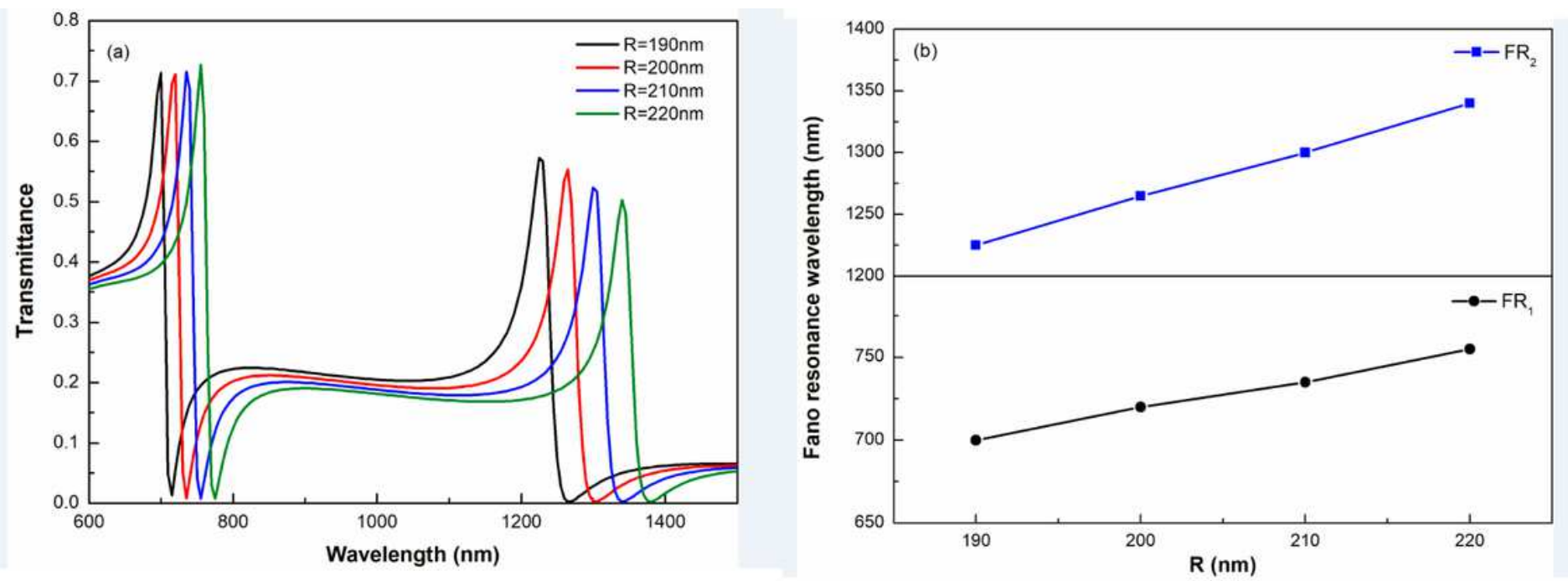

Figure 7

a Transmission spectra of the plasmonic structure with different outer radii $\mathrm{R}, \mathbf{b}$ Relationships between Fano resonance wavelengths and different values of $R$ 
a Transmission spectra of the plasmonic structure with different refractive indices, $\mathbf{b}$ Relationships between Fano resonance wavelengths and different refractive indices

\section{Figure 9}

Sensitivities of the plasmonic structure on $\mathrm{FR}_{1}$ and $\mathrm{FR}_{2}$ for the different parameters at a the coupling distances $\mathrm{g}, \mathbf{b}$ the baffle widths $\mathrm{S}, \mathbf{c}$ the height of rectangular resonator $\mathrm{H}$, and $\mathbf{d}$ the outer radius of the quarter-ring resonator $\mathrm{R}$

Figure 10

FOM for different refractive indices

\section{Figure 11}

a Transmission spectra of the plasmonic structure with the different refractive indices of sucrose solution concentrations, $\mathbf{b}$ Relationships between Fano resonance wavelengths and the refractive indices 